\title{
Review of: "Assessment of four organophosphorus pesticides as inhibitors of human acetylcholinesterase and butyrylcholinesterase"
}

\author{
Slavica Vucinic
}

Potential competing interests: The author(s) declared that no potential competing interests exist.

The aim of the study was to evaluate inhibition of cholinesterase activity with four organophosphorus pesticides both in silico and in vitro with an aim to identify their interactions with active site residues of cholinesterases, as well as the reactivation of pesticide-inhibited AChE with two click quaternary oximes, $1 \mathrm{~A}$ and 14A. These oximes were proven to be the most efficient reactivators of nerve agent tabun-inhibited AChE, which also belongs to the class of phosphoroamidates, same as methamidophos and fenamiphos. In comparison to the standard bispyridinium oximes, it seems that overall reactivation capability of RS194B is limited by lower binding affinity as one could expect for non-permanently charged ligand. It was confirmed that RS194B is a much better reactivator of methamidophos- or fenamiphos-inhibited hAChE than 2-PAM and HI-6 and comparable to obidoxime. This implies that structure of oxime, as well as geometry of oxime approach to the phosphorus atom conjugated to the active serine, are important criteria for efficient reactivation along with the chemical nature of the phosphorus moiety.

The study offers a valuable kinetic data on inhibition of both cholinesterases with four OP pesticides and reactivation of inhibited hAChE.

It is clearly showed that two newly developed reactivators possess excellent potential for further development of antidotes against OP pesticide poisonings. 